\title{
Map Use in Transit Control Centers: Mapping geographic information flows
}

\author{
Emily Domanico
}

Department of Geography, The Pennsylvania State University, exd295@psu.edu

Keywords: Dynamic visualization, Map Use, Control Center, Infrastructure

\begin{abstract}
:
Public transit is an essential part of individual mobility in dense urban environments, and the facilitation of these systems requires the coordination and synchronization of people, objects, and technology through space and time (Fisch 2018). Behind the scenes, control centers are sites where information is reported, synthesized and operationalized. In these environments, maps and diagrams play a central role in the work done to coordinate movement. We present findings from a user-centered study of map use in transit control centers which positions the work done in control centers as a case study for the study of map use in a dynamic, real-time environment. As such, the control center offers an opportunity to study how users navigate the presentation of information interfaces that visualize movement and location information in context of often messy and unpredictable daily workflows.
\end{abstract}

The work of train controllers and dispatchers has changed dramatically with advances in rail signalling, GPS outputs, and automation; however, the study of control center information displays as cartographic products has not kept up with the changing technology (Heath \& Luff 1992). Increasingly, train dispatchers are asked to navigate denser information displays to coordinate the orientation of signals and the safe movement of trains.

We traced how map users understand dynamic, continuously updating visualizations in situ during their daily work. Drawing from ethnographic methods, this study intentionally moves outside of the controlled laboratory, highlighting that maps exist through instances of their use. We constructed a qualitative user study in a three-part methodology that triangulates the process of map use through archival work, participant observation, and survey-based data collection. Further, we developed a body of codes to identify and characterize instances of map use, map communication strategies, and the corresponding information source tied to different behaviors. These codes facilitate the analysis of diverse data sources from descriptions and observations of control centers, interviews with dispatchers, and document analysis. We employed these methods to characterize how map-based interfaces and surrounding technology perform in the socially situated context of the control center where the scope of the map is never limited to an isolated information interface.

The control center is an environment saturated with geographic information. We developed an archive of photographs and videos to characterize the control center environment and identify instances of map use within this space. Inside the modern control center, we find that relative position of train is represented as visual information usually mirrored on large overhead video walls while interaction of the system happens on personal computer monitors (Figure 1). Throughout the
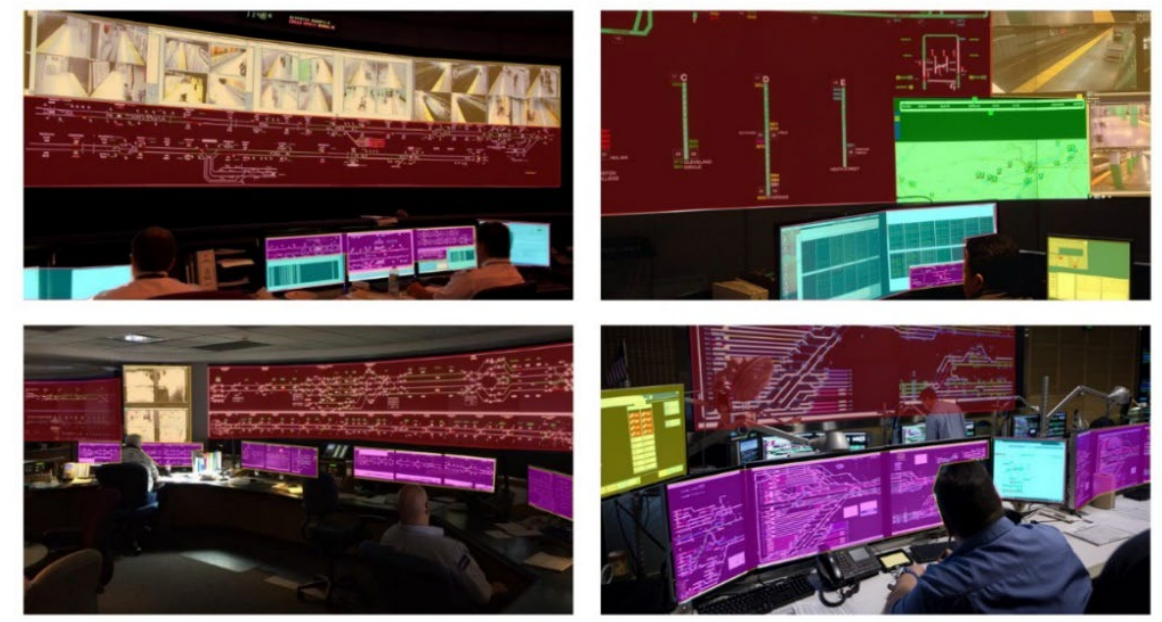

Figure 1. Images from four rail transit control centers with geographic information sources used in rail dispatching highlighted. 
space, schematic line diagrams represent the state of signals throughout the entire rail network, designed to be read at a glance.

Where visual information sources are used to communicate predictable positional data, audio-based communicationsuch as radio to train operators and discussion and planning between dispatchers- supplement what the visual fails to display. Within rail transit visual displays trend to use dynamic, continuously updating schematics to represent states of the transit network, rather than the working history or institutional memory.

Visual schematic maps within the control center prioritize the representation of states of the system rather than an accumulated working history of a day's traffic. The recent history of the daily traffic is pushed onto working memory and verbal communication practices within the dispatching teams which have developed over time. However, the emphasis on visualizing instantaneous states promotes a tendency for dispatchers to view their actions as reflexes rather than strategic responses.

Increasingly, GPS outputs bring more precise real-time train locations in addition to the block-based signaling traditional schematics represent. With the additional information, dispatchers are being asked to work across geographic and schematic representations of space when planning train movements and assessing the speed and flow of trains through the network. Dispatchers identify the importance of aligning information displays and engaging with the information before them as productive for planning efficient maneuvers or spotting unsafe alignments.

Maps and schematics take many forms. It is important for cartographers to study not only how to present geographic information, but also how users respond to these interfaces during the design process but also during the practice of daily use. We argue for the importance of studying maps through instances of their use in context, highlighting the importance of both the physical environment and the social community of users who create and enact the workflows that the geographic displays are designed to support.

Often referred to as 'nerve centers,' control centers are places within transit networks where remotely sensed geographic information is visualized, monitored, and employed to support time-critical decisions. In these environments, maps and diagrams play a central role in the work done to coordinate the safe movements of trains, support staff, and passengers. Within the rail transit control centers, spatial information is presented heterogeneously, where maps take the form of system diagrams, schematic analytical interfaces, layouts of video monitors, and geographic tiled web maps.

As such, we position the control center as a case study for the use of dynamic, real-time, map-based information. Within a control center, daily tasks of train controllers and dispatchers are multiple; they monitor movements, direction, speed, flow, scheduling, and routing, making time-critical decisions at a distance. For the controllers, maps are an interface to the system. We propose that the control center offers a potential space for cartographers to study how users navigate complex, dynamically updating displays that move between abstracted schematics to more traditional geographic maps.

Fisch, M. (2018). An Anthropology of the Machine: Tokyo's Commuter Train Network. Chicago \& London: The University of Chicago Press.

Heath, C. \& P. Luff (1992). "Collaboration and Control: Crisis management and multimedia technology in London Underground Line Control Rooms.” Computer Supported Cooperative Work 1(1-2): 69-94. 